\title{
Téoros
}

Revue de recherche en tourisme

\section{Les comportements touristiques des personnes à capacité physique restreinte au Canada}

\section{Jean Stafford}

Volume 20, numéro 3, automne 2001

Comportements touristiques

URI : https://id.erudit.org/iderudit/1071627ar

DOI : https://doi.org/10.7202/1071627ar

Aller au sommaire du numéro

Éditeur(s)

Université du Québec à Montréal

ISSN

0712-8657 (imprimé)

1923-2705 (numérique)

Découvrir la revue

Citer cet article

Stafford, J. (2001). Les comportements touristiques des personnes à capacité physique restreinte au Canada. Téoros, 20(3), 11-20.

https://doi.org/10.7202/1071627ar d'utilisation que vous pouvez consulter en ligne.

https://apropos.erudit.org/fr/usagers/politique-dutilisation/ 


\section{Les comportements touristiques des personnes à capacité physique restreinte au Canada}

\section{Jean Stafford}

D ans toutes les sociétés développées, le tourisme apparait comme un temps fort de la vie des individus, Malheureusement, pour une bonne partie de la population, les voyages et les vacances semblent inaccessibles. Pour la plupart des personnes qui ne voyagent pas (ou voyagent rarement), le principal obstacle est d'ordre économique. A l'obstacle économique habituel, dans le cas des personnes à capacité physique restreinte s"ajoutent des obstacles physiques.

Depuis plusieurs années, les pouvoirs publics et les organismes communautai= res voués au bien-être des personnes â capacité physique restreinte cherchent à tracer un bilan, le plus exact possible, de la situation. En ce qui concerne l'offre de l'hébergement, de la restauration et du transport, nous avons une image relativement précise de ce qui se passe sur le terrain, mais dans les autres secteurs du tourisme le tableau est un peu plus flou.

Sur le plan de la demande, il n'existait aucunc étude vraiment complète et représentative quant aux départs en vacances et aux comportements touristiques des personnes à capacité physique restreinte. Or, pour aider la compréhension, la prise de décision et les actions à prendre, il était important d'obtenir des données objectives ; l'étude pan-canadienne tente donc de combler cette lacune'.

\section{La méthodologie de I'étude}

La population étudiée est formée des personnes à capacité physique restreinte ${ }^{2}$ au Canada, accessibles par téléphone et pouvant s'exprimer en français ou en anglais. Les entrevues ont été réalisées durant l'année 2000.

Il s'agit d'un échantillon aléatoire, stratifté par provinces (non proportionnel à la taille). Les résultats ont été pondérés (â l'aide des statistiques du recensement de 1996) par le poids démographique de chacune des provinces.

En tenant compte du fait que le poids des provinces est non proportionnel à leur taille, la marge d'erreur maximale théorique sur les réponses, pour les 1003 personnes sondées, est de $\pm 3,1$ \% et ce. pour un intervalle de confiance de $95 \%$. Le taux de réponse est de $65,1 \%$. Le taux d'incidence des personnes à capacité physique restreinte est de l'ordre de 6 à $8 \%$.

\section{Les caractéristiques socio-économiques}

- Les femmes sont légèrement sur-représentées avec 57,5 de l'ensemble.

- En ce qui concerne l'âge, 55 \% de la population interrogée a 55 ans ou plus.

- Les personnes à capacité physique restreinte sont moins scolarisées que l'ensemble de la population globale : il y a une sur-représentation également des personnes avec un niveau primaire ou secondaire, un déficit au niveau collégial et une sur-représentation au niveau universitaire,

- Dans le groupe des personnes à capacité physique restreinte, les $\alpha$ inactifs $n$, c'est-à-dire ceux qui n'ont pas un em- ploi rémunéré, forment $80,3 \%$ de l'ensemble ; les personnes retraitếes représentent $54 \%$ de ces o inactifs $\%$. Les personnes sans emploi ou à la recherche d'un emploi constituent $23 \%$; ce talux de chômage est press du triple de celui de la population canadienne.

- Compte tenu des variables socio-économiques étudiées, les revenus des personnes à capacité physique restreinte sont bas (en comparaison avec la population globale) $; 38 \%$ des répondants gagnent 19999 \$ et moins et $30 \%$ gagnent de $20000 \$$ à 39999 \$. Nous savons, en ce qui concerne les départs en voyages, que le revenu est souvent une wariable déterminante.

- La situation des personnes à capacité physique restreinte varie énormément selon le degré de mobilité ; dans cette étude, nous avons distingué surtout quatre degrés de mobilité:

1 - les personnes qui ont des problèmes d'audition et de vision $(7,4 \%$ de l'échantillon):

2 - les personnes à mobilité réduite (57,1 \% de l'échantillon) :

3 - les personnes à mobilité très réduite (17,6\% de l'échantillon) :

4 - les personnes à mobilité extrêmement réduite $(17,8 \%$ de l'échantillon).

- Les personnes à mobilité teduite font face, souvent, à plusieurs handicaps qui réduisent leur mobilité ; $40 \%$ de ces personnes doivent utiliser un appareil pour leur locomotion ( $26 \%$ une canne ou une marchette, 13 \% un autre appareil et I $\%$ un fauteuil roulant). 
- Dans le groupe des personnes à mobilité très réduite, $63 \%$ utilisent une canne ou une marchette, $12 \%$ un fauteuil roulant et $12 \%$ un autre appareil (ce qui signifie que, dans ce groupe, $67 \%$ utilisent un appareil).

- Enfin, dans le groupe des personnes à mobilité extrêmement réduite, $77 \%$ utilisent un fauteuil roulant, $13 \%$ une canne ou une marchette et $5 \%$ un autre appareil (ainsi, dans ce groupe, $95 \%$ utilisent un appareil).

\section{Le départ en voyage dans la dernière année}

Le cour même de l'étude porte sur le marché touristique des personnes à capacité physique restreinte. Un grand nombre de variables couvrent donc cette dimension importante. La plupart des tableaux portent sur le départ en voyage ainsi que le dernier voyage réalisé au cours de la dernière annéc.

Le tableau | présente les données des départs en voyage d'au moins une nuitée dans la dernière annéc.

Le pourcentage des départs est assez élevé avec $55,7 \%$, mais ce pourcentage n'est pas uniforme; il differe selon les variables socio-economiques usuelles.

Le tableau 2 montre les relations entre les principales variables socio-économiques et certaines variables liées aux voyages réalisés dans la dernière année.

Le tableau 2 permet de constater que toutes les variables socio-économiques choisies influencent le départ en voyage de la dernière année. La destination du demier voyage et le moyen de transport utilise subissent aussi l'influence des variables indếpendantes (six variables sur huit).

Les variables socio-économiques les plus déterminantes semblent être l'occupation, le revenu (avec six sur sept) et aussi, à un degré moindre, l'âge et la scolarité (cinq sur sept). Lä langue maternelle influence quatre variables sur sept. Par ailleurs, le poids des autres variables socio-économiques est marginal.

\begin{tabular}{|c|c|c|}
\hline in: & 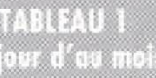 & with \\
\hline Un voyage & Fréquence & $\%$ \\
\hline Oui & 557 & 55,7 \\
\hline Non & 443 & 44,3 \\
\hline TOTAL & 1000 & 100 \\
\hline
\end{tabular}

\begin{tabular}{|c|c|c|c|c|c|c|c|c|}
\hline \multicolumn{9}{|c|}{ 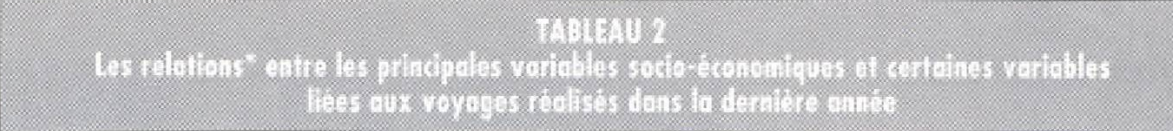 } \\
\hline $\begin{array}{l}\text { Variables liéc: } \\
\text { fux royeges } \\
\text { Variables sucio- } \\
\text { Écomomiques }\end{array}$ & $\begin{array}{l}\text { Voyage } \\
\text { dans la } \\
\text { derniëra } \\
\text { annieb }\end{array}$ & $\begin{array}{l}\text { Nombre de } \\
\text { noprages } \\
\text { dans la } \\
\text { deerière } \\
\text { annege }\end{array}$ & $\begin{array}{l}\text { Nombre de } \\
\text { jours de } \\
\text { dermier } \\
\text { woysge }\end{array}$ & $\begin{array}{l}\text { Destina- } \\
\text { tion du } \\
\text { dernier } \\
\text { vorage }\end{array}$ & $\begin{array}{l}\text { Transpon } \\
\text { lors du } \\
\text { dernier } \\
\text { voyange }\end{array}$ & $\begin{array}{l}\text { Héber- } \\
\text { gemeat } \\
\text { lors du } \\
\text { dernier } \\
\text { vevage }\end{array}$ & $\begin{array}{l}\text { Depenses } \\
\text { lors du } \\
\text { demier } \\
\text { woyage }\end{array}$ & $\begin{array}{l}\text { Total des } \\
\text { relations } \\
\text { significatives }\end{array}$ \\
\hline Mobilité & + & $=$ & - & - & + & - & - & 2 \\
\hline Sexe & + & - & - & - & . & - & - & 1 \\
\hline$\hat{A} g \mathrm{e}$ & + & - & + & + & + & + & . & 5 \\
\hline Région & + & - & $=$ & + & - & . & - & 2 \\
\hline Langue & + & + & - & + & + & . & - & 4 \\
\hline Scolarité & + & + & - & + & + & + & - & 5 \\
\hline Occupation & + & + & + & + & + & + & - & 6 \\
\hline Revenu & + & + & - & + & + & + & + & 6 \\
\hline $\begin{array}{l}\text { Total des } \\
\text { relations } \\
\text { significatives }\end{array}$ & 8 & 4 & 2 & 6 & 6 & 4 & 1 & 31 \\
\hline
\end{tabular}

* Le signe + indique qu'il existe une relation statistiquement significative entre les deux variables pour le test du khi-carré au seuil de 0,10 ou moins.

Le tableau 3 illustre toutes les variables socio-économiques liées au départ en voyage de la dernière année.

Nous constatons, selon les variables socioéconomiques, les pourcentages les plus élevés de départ en voyage dans les catégories suivantes:

- les personnes qui ont des problemes d'audition ou de vision $(66,2 \%)$,

- les hommes $(58,6 \%)$,

- les plus jeunes, 34 ans et moins $(67,3 \%)$,

- dans les provinces des Prairies ou en Colombie-Britannique $\quad(68.5 \%$ et $63,6 \%$ ),
- de langue maternelle anglaise $(61 \%)$

- de niveau universitaire (68\%),

- chez les professionnels et les gestionnaires $(88,5 \%)$,

- dans la strate des revenus les plus élevés, $60000 \$$ et plus $(72,4 \%)$.

Dans certains cas, toujours en ce qui concerne les départs, les différences observées sont très élevées, notamment :

- entre ceux qui ont des problèmes d'audition ou de vision et ceux à mobilité très réduite, l'écart est de $18.2 \%$ :

- entre les plus jeunes et les plus vieux, la différence est de $19,3 \%$; 
- entre les provinces des Prairies et le Québec, l'écart est de 26,7\% ;

- entre les anglophones et les francophones, l'écart est de $17 \%$;

- entre le primaire et le secondaire, la différence est de $26,1 \%$;

- entre les professionnels et gestionnaires et les personnes au foyer, il y a un ecart de $43,9 \%$;

- entre le revenu le plus faible et le revenu le plus élevé, la différence est de $28 \%$.

Il faut souligner qu'entre les hommes et les femmes la différence est minime, soit $5 \%$. Les écarts sont plus accentués entre les 65 ans et plus (un pourcentage de départ de $47,8 \%$ ) et les autres groupes d'âge ; cette dernière remarque est valable aussi pour les francophones et les autres ainsi que pour les gens peu scolarisés (niveau primaire) et ceux qui ont un revenu de $19999 \$$ ou moins.

\section{Le nombre de voyages dans la dernière année}

Le tableau 4 présente le nombre de voyages dans la dernière année.

On peut constater que $53,6 \%$ des personnes interrogées font deux voyages et moins par année alors que $21,3 \%$ en font 6 ou plus par année.

Au tableau 5, nous présentons les relations entre le nombre de woyages effectués dans l'année et certaines variables indépendantes; les relations avec les autres variables socio-économiques ne sont pas statistiquement significatives.

Ce sont les personnes de langue maternelle « autres \& qui voyagent le moins souvent dans la même année : 70,2 \% d'entre elles ne partent qu'une ou deux fois. Ce pourcentage est de $50,4 \%$ chez les anglophones et de $51,7 \%$ chez les francophones.

Les personnes qui ont une scolarité de niveau primaire ou de niveau secondaire voyagent moins souvent que celles qui ont une scolarité de niveau collégial ou de niveau universitaire. Les personnes sans emploi ct ayant un revenu égal ou inférieur

\begin{tabular}{|c|c|c|c|c|}
\hline \multicolumn{5}{|c|}{ 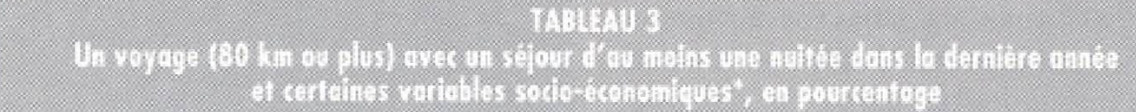 } \\
\hline \multicolumn{2}{|r|}{ Variables } & \multirow{5}{*}{$\begin{array}{l}\text { 0ui } \\
66,2 \\
58,1 \\
48,0 \\
51,1\end{array}$} & \multirow{5}{*}{$\begin{array}{l}\text { Non } \\
33,8 \\
41,9 \\
52,0 \\
48,9\end{array}$} & \multirow{5}{*}{$\begin{array}{c}\text { Total } \\
100 \\
100 \\
100 \\
100\end{array}$} \\
\hline Mobilité & Audition ou vision & & & \\
\hline & Mobilité réduite & & & \\
\hline & Mobilité très réduite & & & \\
\hline & Mobilité extrêmement réduite & & & \\
\hline \multirow[t]{2}{*}{ Sexe } & Masculin & 58,6 & 41,4 & 100 \\
\hline & Fêminin & 53,6 & 46,4 & 100 \\
\hline \multirow[t]{6}{*}{$\hat{A} g e$} & 24 ans et moins & 67,1 & 32,9 & 100 \\
\hline & $25-34$ ans & 67,3 & 32,7 & 100 \\
\hline & $35-44$ ans & 60,8 & 39,2 & 100 \\
\hline & $45-54$ ans & 59.9 & 40,1 & 100 \\
\hline & $55-64$ ans & 56,2 & 43,8 & 100 \\
\hline & 65 ans et plus & 47,8 & 52,2 & 100 \\
\hline \multirow[t]{5}{*}{ Région } & Maritimes & 54,4 & 45,6 & 100 \\
\hline & Québec & 41,8 & 58,2 & 100 \\
\hline & Ontario & 56,3 & 43,7 & 100 \\
\hline & Prairies & 68,5 & 31,5 & 100 \\
\hline & Colombie-Britannique & 63,6 & 36,4 & 100 \\
\hline \multirow[t]{3}{*}{ Langue } & Français & 44,0 & 56,0 & 100 \\
\hline & Anglais & 61,0 & 39,0 & 100 \\
\hline & Autres & 58,0 & 42,0 & 100 \\
\hline \multirow[t]{4}{*}{ Scolarité } & Primaire & 41,9 & 58,1 & 100 \\
\hline & Secondaire & 53,9 & 46,1 & 100 \\
\hline & Collegial & 61,0 & 39,0 & 100 \\
\hline & Universitaire & 68,0 & 32,0 & 100 \\
\hline \multirow[t]{7}{*}{ Occupation } & Professionnels/gestionnaires & 88,5 & 11,5 & 100 \\
\hline & Services & 73,8 & 26,2 & 100 \\
\hline & Techniciens/ouvriers & 65,6 & 34,4 & 100 \\
\hline & Au foyer & 44,6 & 55,4 & 100 \\
\hline & Étudiants & 65,3 & 34,7 & 100 \\
\hline & Retraités & 50,0 & 50,0 & 100 \\
\hline & Sans emploi & 54,9 & 45,1 & 100 \\
\hline \multirow[t]{4}{*}{ Revenu total } & 19999 \$ et moins & 44,7 & 55,3 & 100 \\
\hline & $20000 \$-39999 \$$ & 55,1 & 44,9 & 100 \\
\hline & $40000 \$-59999 \$$ & 70,5 & 29,5 & 100 \\
\hline & 60000 \$ et plus & 72,4 & 27,6 & 100 \\
\hline
\end{tabular}

* Toutes les relations entre les variables présentées dans ce tableau sont statistiquement significatives (le test du khi-carré) au seuil de 0,10 ou moins.

\begin{tabular}{|c|c|c|c|}
\hline \multicolumn{4}{|c|}{ (o. } \\
\hline Nambre de voyages & Fréquence & $\%$ & \% cumulé \\
\hline 1 fois & 157 & 29,8 & 29,8 \\
\hline 2 fois & 126 & 23,8 & 53,6 \\
\hline $3-5$ fois & 132 & 25,1 & 78,7 \\
\hline 6 fois et plus & 113 & 21,3 & 100 \\
\hline TOTAL & 528 & 100 & \\
\hline
\end{tabular}


à 19999 \$ voyagent moins souvent que les autres dans la même année. On constate donc que la langue parlée, la scolarité, l'occupation principale et le revenu sont des variables déterminantes sur la fréquence des voyages annuels,

\section{La durée des séjours lors du dernier voyage}

Le tableau 6 montre le nombre de jours de vacances lors du dernier voyage.

Nous constatons à la lecture de ce tableau que $73,3 \%$ des répondants prennent une semaine ou moins lors du dernier voyage réalisé́.

Le tableau 7 présente les relations entre le nombre de jours de voyages et deux variables indépendantes : l'âge et l'occupation principale. La relation avec les autres variables socio-économiques n'est pas significative.

Ce sont les personnes de 24 ans et moins et les personnes de 55 ans et plus qui font les plus longs voyages (plus d'une semaine). Pour ce qui est de la variable occupation, ce sont les étudiants, les retraités et les personnes qui travaillent dans le secteur des services qui voyagent le plus longtemps.

\section{La destination principale du dernier voyage réalisé}

Les principales destinations du dernier voyage apparaissent au tableau 8 .

Près de $27,7 \%$ des personnes à capacité physique restreinte voyagent à l'extérieur' du Canada: $15.1 \%$ aux États-Unis et $12,6 \%$ dans d'autres pays. Le tableau 9 présente les relations entre la destination principale el six variables indépendantes qui influencent le choix de la destination.

Ce sont les personnes situécs aux extrémités de l'échelle des âges qui voyagent le plus à l'étranger (États-Unis et autres pays). Le groupe des 55-64 ans remporte la palme avec $36,2 \%$ de voyages à l'étranger.

\begin{tabular}{|c|c|c|c|c|c|c|}
\hline \multicolumn{7}{|c|}{ 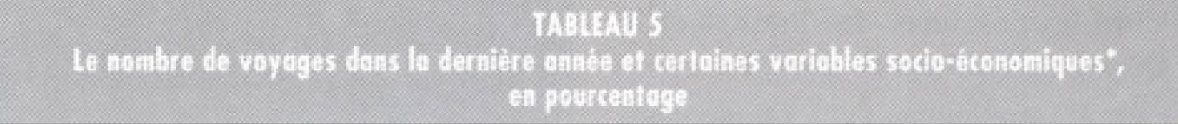 } \\
\hline \multicolumn{2}{|r|}{ Variables } & 1 fois & 2 fois & $\begin{array}{l}3-5 \text { fois } \\
\text { et plus }\end{array}$ & 6 fois & Total \\
\hline Langue & $\begin{array}{l}\text { Français } \\
\text { Anglais } \\
\text { Autres }\end{array}$ & $\begin{array}{l}25,4 \\
27,2 \\
48,1\end{array}$ & $\begin{array}{l}26,3 \\
23,3 \\
22,1\end{array}$ & $\begin{array}{l}25,4 \\
27,2 \\
15,6\end{array}$ & $\begin{array}{l}22,9 \\
22,4 \\
14,2\end{array}$ & $\begin{array}{l}100 \\
100 \\
100\end{array}$ \\
\hline Scolarité & $\begin{array}{l}\text { Primaire } \\
\text { Secondaire } \\
\text { Collegial } \\
\text { Universitaire }\end{array}$ & $\begin{array}{l}34,5 \\
34,0 \\
24,7 \\
20,4\end{array}$ & $\begin{array}{l}22,4 \\
25,7 \\
21,6 \\
23,0\end{array}$ & $\begin{array}{l}15,5 \\
23,7 \\
30,9 \\
28,3\end{array}$ & $\begin{array}{l}27,6 \\
16,6 \\
22,8 \\
28,3\end{array}$ & $\begin{array}{l}100 \\
100 \\
100 \\
100\end{array}$ \\
\hline Occupation & $\begin{array}{l}\text { Professionnels/gestionnaires } \\
\text { Services } \\
\text { Techniciens/ouvriers } \\
\text { Au foyer } \\
\text { Étudiants } \\
\text { Retraités } \\
\text { Sans emploi }\end{array}$ & $\begin{array}{r}9,1 \\
34,4 \\
31,4 \\
27,8 \\
16,7 \\
32,0 \\
35,4\end{array}$ & $\begin{array}{l}15,9 \\
29,5 \\
22,9 \\
23,0 \\
26,7 \\
24,1 \\
24,0\end{array}$ & $\begin{array}{l}34,1 \\
21,3 \\
22,9 \\
24,6 \\
23,3 \\
25,6 \\
24,0\end{array}$ & $\begin{array}{l}40,9 \\
14,8 \\
22,8 \\
24,6 \\
33,3 \\
18,3 \\
16,7\end{array}$ & $\begin{array}{l}100 \\
100 \\
100 \\
100 \\
100 \\
100 \\
100\end{array}$ \\
\hline Revenu total & $\begin{array}{l}19999 \text { \$ et moins } \\
20000 \text { \$-39 } 999 \$ \\
40000 \text { \$-59 } 999 \text { \$ } \\
60000 \text { \$ et plus }\end{array}$ & $\begin{array}{l}39,7 \\
30,3 \\
22,7 \\
16,3\end{array}$ & $\begin{array}{l}27,8 \\
16,4 \\
27,3 \\
23,6\end{array}$ & $\begin{array}{l}21,4 \\
32,0 \\
26,1 \\
26,7\end{array}$ & $\begin{array}{l}11,1 \\
21,3 \\
23,9 \\
20,9\end{array}$ & $\begin{array}{l}100 \\
100 \\
100 \\
100\end{array}$ \\
\hline
\end{tabular}

* Toutes les relations entre les variables présentées dans ce tableau sont statistiquement significatives (le test du khi-carré) au seuil de 0,10 ou moins.

\begin{tabular}{|lccc|}
\hline \multicolumn{3}{c|}{ TAminat 6} \\
\hline Nombre de jours & Fréquence & $\%$ & \% cumule \\
\hline $1-2$ jours & 156 & 28,6 & 28,6 \\
$3-4$ jours & 144 & 26,5 & 55,1 \\
$5-7$ jours & 100 & 18,3 & 73,3 \\
$8-14$ jours & 79 & 14,4 & 87,7 \\
15 jours et plus & 67 & 12,3 & 100 \\
TOTAL & 545 & 100 & \\
\hline
\end{tabular}

\begin{tabular}{|c|c|c|c|c|c|c|c|}
\hline \multicolumn{8}{|c|}{ 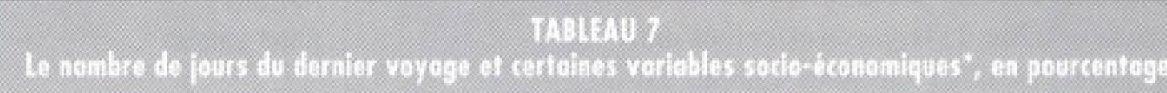 } \\
\hline & Variables & $\begin{array}{c}1-2 \\
\text { jours }\end{array}$ & $\begin{array}{c}3-4 \\
\text { jours }\end{array}$ & $\begin{array}{l}\text { 5-7 } \\
\text { jours }\end{array}$ & $\begin{array}{l}8-14 \\
\text { jours }\end{array}$ & $\begin{array}{l}15 \text { jours } \\
\text { et plus }\end{array}$ & Total \\
\hline Âge & $\begin{array}{l}24 \text { ans et moins } \\
25-34 \text { ans } \\
35-44 \text { ans } \\
45-54 \text { ans } \\
55-64 \text { ans } \\
65 \text { ans et plus }\end{array}$ & $\begin{array}{l}18,5 \\
29,7 \\
36,5 \\
29,7 \\
30,8 \\
25,5\end{array}$ & $\begin{array}{l}27,8 \\
24,3 \\
29,7 \\
36,0 \\
23,1 \\
21,8\end{array}$ & $\begin{array}{l}24,0 \\
21,6 \\
18,9 \\
17,1 \\
16,3 \\
17,0\end{array}$ & $\begin{array}{c}16,7 \\
16,2 \\
10,8 \\
9,1 \\
18,3 \\
16,4\end{array}$ & $\begin{array}{c}13,0 \\
8,1 \\
4,1 \\
8,1 \\
11,5 \\
19,4\end{array}$ & $\begin{array}{l}100 \\
100 \\
100 \\
100 \\
100 \\
100\end{array}$ \\
\hline Qccupation & $\begin{array}{l}\text { Professionnels/ } \\
\text { gestionnaires } \\
\text { Services } \\
\text { Techniciens/ouvriers } \\
\text { Au foyer } \\
\text { Etudiants } \\
\text { Retraites } \\
\text { Sans emploi }\end{array}$ & $\begin{array}{l}26,7 \\
16,4 \\
17,5 \\
42,6 \\
22,6 \\
27,5 \\
37,0\end{array}$ & $\begin{array}{l}33,3 \\
32,8 \\
25,0 \\
27,9 \\
19,4 \\
23,2 \\
27,0\end{array}$ & $\begin{array}{l}15,5 \\
19,6 \\
40,0 \\
11,5 \\
25,8 \\
16,9 \\
15,0\end{array}$ & $\begin{array}{r}17,8 \\
14,8 \\
12,5 \\
16,4 \\
16,1 \\
16,5 \\
9,0\end{array}$ & $\begin{array}{c}6,7 \\
16,4 \\
5,0 \\
1,6 \\
16,1 \\
15,9 \\
12,0\end{array}$ & $\begin{array}{l}100 \\
100 \\
100 \\
100 \\
100 \\
100 \\
100\end{array}$ \\
\hline
\end{tabular}

* Toutes les relations entre les variables présentées dans ce tableau sont statistiquement significatives (le test du khi-carré) au seuil de 0,10 ou moins. 
Sur le plan régional, ce sont les habitants de l'Ontario $(32,8 \%)$ et ceux de la Colombie-Britannique $(37,8 \%)$ qui voyagent le plus à l'étranger. La langue maternelle a aussi une influence puisque $28,9 \%$ des anglophones (contre seulement 14,4\% des francophones), et $43,7 \%$ de ceux dont la langue maternelle n'est ni le français ni l'anglais, voyagent à l'étranger (lors de leur dernier voyage).

Les voyages aux États-Unis sont plus nombreux chez les personnes ayant une scolarité de niveau collégial $(18,2 \%)$ ou universitaire $(21,1 \%)$. Les voyages âl l'étranger, â l'exception des Etats-Unis, sont plus fréquents chez les personnes ayant des études secondaires ou collégiales.

Ce sont les professionnels et les gestionnaires qui woyagent le plus aux États-Unis $(28,9 \%)$ alors que ce sont les employés des services qui voyagent le plus à l'étranger $(23 \%)$, dans d'autres pays que les États-Unis. Les voyages aux États-Unis augmentent généralement avec les revenus ; dans le cas des voyages à l'étranger (à l'exception des États-Unis), il y a une nette distinction entre ceux qui gagnent 39999 \$ et moins et ceux qui gagnent 40000 \$ et plus (le pourcentage double dans cette dernière catégorie de revenu).

\section{Le moyen de transport lors du dernier voyage}

Le tableau 10 fait état du moyen de transport utilisé lors du dernier voyage.

L'automobile est le moyen de transport le plus utilisé par $62,6 \%$ des répondants ; l'avion suit avec 19,1\%. Les autres modes de transport montrent des pourcentages moindres.

Au tableau 11, nous présentons les relations entre le moyen de transport utilisé lors du dernier voyage et certaines variables socio-économiques.

Le choix du mode de transport varie quelque peu selon le degré de mobilité du répondant. L'automobile est le moyen de transport le plus utilisé, mais les fréquences. sont différentes selon le degré de mobilité :

\begin{tabular}{|c|c|c|}
\hline \multicolumn{3}{|c|}{ 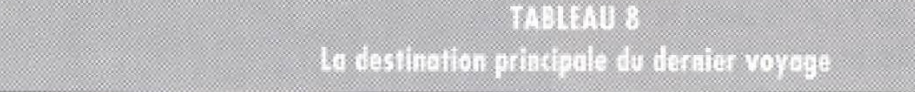 } \\
\hline Destination & Fréquence & $\%$ \\
\hline Québec & 85 & 15,5 \\
\hline Ontario & 144 & 26,1 \\
\hline Ouest Canada & 140 & 25,3 \\
\hline Est Canada & 30 & 5,4 \\
\hline États-Unis & 84 & 15,1 \\
\hline Autres & 70 & 12,6 \\
\hline TOTAL & 552 & 100 \\
\hline
\end{tabular}

\begin{tabular}{|c|c|c|c|c|c|c|c|c|}
\hline \multicolumn{9}{|c|}{ 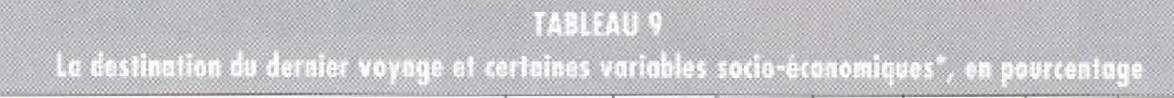 } \\
\hline & Variables & Québec & Ontaria & $\begin{array}{c}\text { Duest } \\
\text { Canada }\end{array}$ & $\begin{array}{c}\text { Est } \\
\text { Canada }\end{array}$ & Etats-Unis & Autres & Total \\
\hline Âge & $\begin{array}{l}24 \text { ans et moins } \\
25-34 \text { ans } \\
35-44 \text { ans } \\
45-54 \text { ans } \\
55-64 \text { ans } \\
65 \text { ans et plus }\end{array}$ & $\begin{array}{c}21,2 \\
13,5 \\
20,2 \\
19,3 \\
6,7 \\
15,2\end{array}$ & $\begin{array}{l}21,2 \\
29,7 \\
37,3 \\
21,1 \\
29,5 \\
23,4\end{array}$ & $\begin{array}{l}13,5 \\
18,9 \\
20,0 \\
34,9 \\
23,8 \\
26,9\end{array}$ & $\begin{array}{l}13,5 \\
5,4 \\
4,0 \\
6,4 \\
3,8 \\
4,1\end{array}$ & $\begin{array}{c}15,4 \\
16,2 \\
9,3 \\
11,0 \\
20,0 \\
17,0\end{array}$ & $\begin{array}{c}15,2 \\
16,3 \\
9,4 \\
7,3 \\
16,2 \\
13,4\end{array}$ & $\begin{array}{l}100 \\
100 \\
100 \\
100 \\
100 \\
100\end{array}$ \\
\hline Région & $\begin{array}{l}\text { Maritimes } \\
\text { Québec } \\
\text { Ontario } \\
\text { Prairies } \\
\text { Colombie-Britannique }\end{array}$ & $\begin{array}{l}11,9 \\
66,3 \\
5,1 \\
0,9 \\
1,2\end{array}$ & $\begin{array}{c}14,3 \\
9,9 \\
55,1 \\
3,5 \\
6,1\end{array}$ & $\begin{array}{c}4,8 \\
0,0 \\
5,6 \\
71,1 \\
54,9\end{array}$ & $\begin{array}{l}52,4 \\
4,0 \\
1,4 \\
0,9 \\
0,0\end{array}$ & $\begin{array}{l}9,5 \\
12,9 \\
17,8 \\
14,0 \\
17,1\end{array}$ & $\begin{array}{c}7,1 \\
6,9 \\
15,0 \\
9,6 \\
20,7\end{array}$ & $\begin{array}{l}100 \\
100 \\
100 \\
100 \\
100\end{array}$ \\
\hline Langue & $\begin{array}{l}\text { Français } \\
\text { Anglais } \\
\text { Autres }\end{array}$ & $\begin{array}{l}56,6 \\
2,6 \\
6,3\end{array}$ & $\begin{array}{l}14,5 \\
31,5 \\
20,0\end{array}$ & $\begin{array}{c}8,1 \\
30,6 \\
30,0\end{array}$ & $\begin{array}{l}6,5 \\
6,4 \\
0,0\end{array}$ & $\begin{array}{c}9,7 \\
13,6 \\
31,3\end{array}$ & $\begin{array}{c}4,7 \\
15,3 \\
12,4\end{array}$ & $\begin{array}{l}100 \\
100 \\
100\end{array}$ \\
\hline Scolarité & $\begin{array}{l}\text { Primaire } \\
\text { Secondaire } \\
\text { Collégial } \\
\text { Universitaire }\end{array}$ & $\begin{array}{l}29,0 \\
13,4 \\
10,1 \\
15,8\end{array}$ & $\begin{array}{l}22,6 \\
27,6 \\
28,3 \\
23,7\end{array}$ & $\begin{array}{l}17,7 \\
28,0 \\
24,2 \\
25,4\end{array}$ & $\begin{array}{l}11,3 \\
4,1 \\
5,1 \\
5,3\end{array}$ & $\begin{array}{l}12,9 \\
11,9 \\
18,2 \\
21,1\end{array}$ & $\begin{array}{c}6,5 \\
15,0 \\
14,1 \\
8,6\end{array}$ & $\begin{array}{l}100 \\
100 \\
100 \\
100\end{array}$ \\
\hline Occupation & $\begin{array}{l}\text { Professionnels! } \\
\text { gestionnaires } \\
\text { Services } \\
\text { Techniciens/ouvriers } \\
\text { Au foyer } \\
\text { Etudiants } \\
\text { Retraités } \\
\text { Sans emploi }\end{array}$ & $\begin{array}{l}13,3 \\
16,4 \\
13,2 \\
16,4 \\
28,0 \\
15,0 \\
13,0\end{array}$ & $\begin{array}{l}20,0 \\
23,0 \\
28,9 \\
27,9 \\
18,8 \\
25,4 \\
32,0\end{array}$ & $\begin{array}{l}22,2 \\
19,6 \\
23,7 \\
27,9 \\
18,8 \\
27,2 \\
28,0\end{array}$ & $\begin{array}{c}2,2 \\
\\
1,6 \\
5,3 \\
9,8 \\
18,8 \\
3,3 \\
6,0\end{array}$ & $\begin{array}{c}28,9 \\
16,4 \\
15,8 \\
13,1 \\
15,6 \\
15,5 \\
9,0\end{array}$ & $\begin{array}{c}13,4 \\
\\
23,0 \\
13,1 \\
4,9 \\
0,0 \\
13,6 \\
12,0\end{array}$ & $\begin{array}{l}100 \\
100 \\
100 \\
100 \\
100 \\
100 \\
100\end{array}$ \\
\hline Revenu total & $\begin{array}{l}19999 \$ \text { et moins } \\
20000 \$-39999 \$ \\
40000 \$-59999 \$ \\
60000 \$ \text { et plus }\end{array}$ & $\begin{array}{l}14,7 \\
14,8 \\
18,5 \\
14,3\end{array}$ & $\begin{array}{l}32,6 \\
32,8 \\
17,4 \\
27,4\end{array}$ & $\begin{array}{l}31,8 \\
21,9 \\
21,7 \\
20,2\end{array}$ & $\begin{array}{l}4,7 \\
7,0 \\
4,3 \\
3,6\end{array}$ & $\begin{array}{l}9,2 \\
16,4 \\
19,6 \\
15,5\end{array}$ & $\begin{array}{l}7,0 \\
7,1 \\
18,5 \\
19,0\end{array}$ & $\begin{array}{l}100 \\
100 \\
100 \\
100\end{array}$ \\
\hline
\end{tabular}

* Toutes les relations entre les variables présentées dans ce tableau sont statistiquement significatives lle test du khi-carré) au seuil de 0,10 ou moins. 
le pourcentage est de $71,4 \%$ dans le cas de mobilité très réduite, de $51,6 \%$ pour une mobilité extrêmement réduite et de $51 \%$ pour les personnes qui ont un problème d'audition ou de vision.

L'âge a une certaine incidence dans l'utilisation de l'autobus comme moyen de transport : $18,4 \%$ des $25-34$ ans utilisent ce moyen de transport et le pourcentage d"utilisation est beaucoup plus faible pour les autres groupes d'âge. Ce sont les jeunes de 24 ans et moins qui ont le pourcentage le plus élevé $(22,6 \%)$ d'utilisation d'un moyen de transport différent de l'avion, de l'automobile ou de l'autobus.

L'utilisation de l'avion varie assez fortement selon la langue maternelle : $10,5 \%$ chez les francophones, $18,8 \%$ chez les anglophones et $34,2 \%$ pour les autres langues. On remarque aussi, au tableau II, que ce sont les personnes de niveau collégial qui voyagent le moins en avion $(9 \%$ comparé a $21,9 \%$ dans les autres catégories). Pour toutes les catégories d'occupation l'avion et l'automobile sont les moyens de transport les plus utilisés. Les catégories des services et des techniciens et ouvriers sont plus nombreuses à utiliser l'autobus ou d'autres moyens de transport que les autres catégories dejà citées. L"utilisation de l'avion augmente très légèrement avec l'augmentation des revenus, alors que l'on observe le phénomène inverse avec l'utilisation de l'autobus.

\section{Le mode d'hébergement lors du dernier voyage}

Le mode d'hébergement choisi, lors du dernier voyage, est démontré au tableau 12 .

Ce sont les séjours chez des parents et des amis qui correspondent au mode d'hébergement préféré $(47,3 \%)$; l'hébergement à l'hôtel ou au motel arrive en deuxième position avec $30,8 \%$. Les autres modes privés d'hébergement recueillent $21,9 \%$ des choix des répondants. Il semble que les personnes à capacité physique restreinte utilisent, plus que les autres canadiens, les hôtels et les motels ( $19 \%$ des nuitéespersonnes des Canadiens en $1998^{3}$ ). Quant à l'hebergement chez des parents ou des

\begin{tabular}{|l|c|c|}
\hline \multicolumn{3}{|c|}{ Tamizmi 10} \\
\hline Moyen de transport & Fréquence & $\%$ \\
\hline Avion & 106 & 19,1 \\
Automobile & 349 & 62,6 \\
Autobus & 40 & 7,1 \\
Autres & 63 & 11,3 \\
Total & 557 & 100 \\
\hline
\end{tabular}

\begin{tabular}{|c|c|c|c|c|c|c|}
\hline \multicolumn{7}{|c|}{ 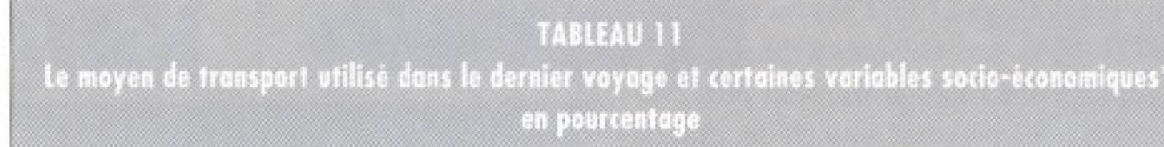 } \\
\hline & Variables & Avion & Auto & Autobus & Autres & Total \\
\hline Mobilité & $\begin{array}{l}\text { Audition ou vision } \\
\text { Mobilité réduite } \\
\text { Mobilité très réduite } \\
\text { Mobilité extrêment réduite }\end{array}$ & $\begin{array}{l}16,3 \\
19,6 \\
16,7 \\
20,9\end{array}$ & $\begin{array}{l}51,0 \\
65,1 \\
71,4 \\
51,6\end{array}$ & $\begin{array}{l}14,3 \\
5,7 \\
4,8 \\
9,9\end{array}$ & $\begin{array}{c}18,4 \\
9,6 \\
7,1 \\
17,6\end{array}$ & $\begin{array}{l}100 \\
100 \\
100 \\
100\end{array}$ \\
\hline Äge & $\begin{array}{l}24 \text { ans et moins } \\
25-34 \text { ans } \\
35-44 \text { ans } \\
45-54 \text { ans } \\
55-64 \text { ans } \\
65 \text { ans et plus }\end{array}$ & $\begin{array}{l}13,2 \\
18,4 \\
15,8 \\
14,3 \\
21,2 \\
24,0\end{array}$ & $\begin{array}{l}58,5 \\
60,5 \\
63,2 \\
68,8 \\
58,7 \\
62,9\end{array}$ & $\begin{array}{c}5,7 \\
18,4 \\
9,2 \\
3,6 \\
7,7 \\
6,9\end{array}$ & $\begin{array}{c}22,6 \\
2,7 \\
11,8 \\
13,3 \\
12,5 \\
6,3\end{array}$ & $\begin{array}{r}100 \\
100 \\
100 \\
100 \\
100 \\
100\end{array}$ \\
\hline Langue & $\begin{array}{l}\text { Français } \\
\text { Anglais } \\
\text { Autres }\end{array}$ & $\begin{array}{l}10,5 \\
18,8 \\
34,2\end{array}$ & $\begin{array}{l}65,3 \\
62,5 \\
58,2\end{array}$ & $\begin{array}{l}9,7 \\
7,1 \\
2,5\end{array}$ & $\begin{array}{c}14,5 \\
11,6 \\
5,1\end{array}$ & $\begin{array}{l}100 \\
100 \\
100\end{array}$ \\
\hline Scolarité & $\begin{array}{l}\text { Primaire } \\
\text { Secondaire } \\
\text { Collégial } \\
\text { Universitaire }\end{array}$ & $\begin{array}{c}22,6 \\
20,8 \\
9,0 \\
22,4\end{array}$ & $\begin{array}{l}53,2 \\
64,4 \\
64,0 \\
61,2\end{array}$ & $\begin{array}{c}8,1 \\
5,2 \\
10,0 \\
7,8\end{array}$ & $\begin{array}{c}16,1 \\
9,6 \\
17,0 \\
8,6\end{array}$ & $\begin{array}{l}100 \\
100 \\
100 \\
100\end{array}$ \\
\hline Occupation & $\begin{array}{l}\text { Professionnels/ } \\
\text { gestionnaires } \\
\text { Services } \\
\text { Techniciens/ouvriers } \\
\text { Au foyer } \\
\text { Étudiants } \\
\text { Retraités } \\
\text { Sans emploi }\end{array}$ & $\begin{array}{l}21,7 \\
21,0 \\
17,5 \\
11,3 \\
25,0 \\
22,3 \\
12,9\end{array}$ & $\begin{array}{l}76,1 \\
53,2 \\
52,5 \\
66,1 \\
53,1 \\
61,9 \\
68,3\end{array}$ & $\begin{array}{c}2,2 \\
14,5 \\
10,0 \\
6,5 \\
9,4 \\
6,0 \\
5,9\end{array}$ & $\begin{array}{r}0,0 \\
11,3 \\
20,0 \\
16,1 \\
12,5 \\
9,8 \\
12,9\end{array}$ & $\begin{array}{l}100 \\
100 \\
100 \\
100 \\
100 \\
100\end{array}$ \\
\hline Revenu total & $\begin{array}{l}19999 \text { \$ et moins } \\
20000 \$-39999 \$ \\
40000 \$-59999 \$ \\
60000 \text { \$ et plus }\end{array}$ & $\begin{array}{l}15,3 \\
12,5 \\
22,6 \\
22,9\end{array}$ & $\begin{array}{l}62,6 \\
66,4 \\
65,6 \\
60,2\end{array}$ & $\begin{array}{l}13,7 \\
6,3 \\
4,3 \\
3,6\end{array}$ & $\begin{array}{c}8,4 \\
14,8 \\
7,5 \\
13,3\end{array}$ & $\begin{array}{l}100 \\
100 \\
100 \\
100\end{array}$ \\
\hline
\end{tabular}

* Toutes les relations entre les variables présentées dans ce tableau sont statistiquement significatives (le test du khi-carré) au seuil de 0,10 ou moins. 
amis, nous constatons à peu prè̀ les mêmes pourcentages ( $49 \%$ des nuitées-personnes) dans la population canadienne.

Le tableau 13 présente le choix du mode d'hébergement lié à quatre variables indépendantes.

Le groupe des 25-44 ans privilégie plus fortement que les autres groupes le choix de l'hotel ou du motel. Les autres modes privés d'hébergement ont surtout la faveur des plus jeunes (avec $36,5 \%$ ), alors que les 45 54 ans et le groupe des 65 ans et plus séjournent plutôt chez des parents ou des amis.

Le séjour à l'hôtel ou au motel augmente fortement avec le degré de scolarité $(19,7 \%$ au primaire et $43,5 \%$ au niveau universitaire). Il y a aussi une différence nette dans l'utilisation de l'hôtel ou du motel comme mode d'hébergement et l'occupation principale; ici il y a un écart entre les «actifs * (ceux qui ont un emploi)et les $\alpha$ inactifs $»$ (ceux qui n'ont pas d'emploi rémunéré), Le mode d'hébergement chez les parents et les amis est très élevé chez les personnes au foyer, les retraités et les sans emploi. Le choix de l'hôtel ou du motel comme mode d'hébergement augmente avec la strate des revenus $(22,5 \%$ dans la strate des $19999 \$$ et moins et $47,6 \%$ dans la strate des $60000 \$$ et plus). Nous constatons la situation inverse dans le mode de séjour chez des parents et des amis.

\section{Les dépenses lors du dernier voyage}

Le tableau 14 fait état des dépenses effectuées lors du dernier voyage.

Nous constatons à la lecture de ce tableau que $74,5 \%$ des personnes interrogées dépensent $500 \$$ ou moins lors de leur dernier séjour de vacances.

Les dépenses varient fortement selon les revenus des répondants. Le lien entre les dépenses et les revenus est démontré au tableau 15.

Dans les catégories $501 \$$ à $2000 \$$ et $2001 \$$ et plus, les dépenses augmentent

\begin{tabular}{|l|c|c|}
\hline \multicolumn{2}{|c|}{} \\
\hline Mode d'héhergement & Fréquence & $\%$ \\
\hline Hotel/Motel & 170 & 30,8 \\
Bed and Breakfast/Gîte & 7 & 1,3 \\
Camping/Caravaning & 25 & 4,5 \\
Chalet/Condotel & 23 & 4,1 \\
Camp de vacances/Auberges de jeuness & 5 & 0,9 \\
Parents/Amis & 262 & 47,3 \\
Autres endroits & 61 & 11,1 \\
TOTAL & 553 & 100 \\
\hline
\end{tabular}

\begin{tabular}{|c|c|c|c|c|c|}
\hline \multicolumn{6}{|c|}{ 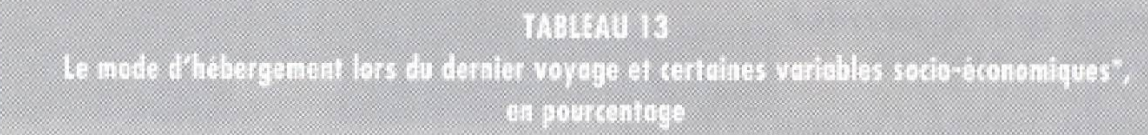 } \\
\hline & Variables & Hibtel! & Autres modes & Parentsif & Total \\
\hline Âge & $\begin{array}{l}24 \text { ans et moins } \\
25-34 \text { ans } \\
35-44 \text { ans } \\
45-54 \text { ans } \\
55-64 \text { ans } \\
65 \text { ans et plus }\end{array}$ & $\begin{array}{l}26,9 \\
43,2 \\
41,3 \\
26,5 \\
35,6 \\
23,8\end{array}$ & $\begin{array}{c}36,5 \\
8,1 \\
21,3 \\
17,7 \\
24,0 \\
22,1\end{array}$ & $\begin{array}{l}36,7 \\
48,7 \\
37,4 \\
55,8 \\
40,4 \\
54,1\end{array}$ & $\begin{array}{l}100 \\
100 \\
100 \\
100 \\
100 \\
100\end{array}$ \\
\hline Scolarité & $\begin{array}{l}\text { Primaire } \\
\text { Secondaire } \\
\text { Collégial } \\
\text { Universitaire }\end{array}$ & $\begin{array}{l}19,7 \\
26,8 \\
36,7 \\
43,5\end{array}$ & $\begin{array}{l}29,5 \\
22,3 \\
19,4 \\
19,1\end{array}$ & $\begin{array}{l}50,8 \\
50,9 \\
43,9 \\
37,4\end{array}$ & $\begin{array}{r}100 \\
100 \\
100 \\
100\end{array}$ \\
\hline Occupation & $\begin{array}{l}\text { Professionnels/gestionnaires } \\
\text { Services } \\
\text { Techniciens/ouvriers } \\
\text { Au foyer } \\
\text { Étudiants } \\
\text { Retraités } \\
\text { Sans emploi }\end{array}$ & $\begin{array}{l}52,2 \\
41,9 \\
42,5 \\
24,6 \\
34,4 \\
26,0 \\
22,4\end{array}$ & $\begin{array}{l}13,0 \\
19,4 \\
22,5 \\
18,0 \\
37,5 \\
21,4 \\
25,5\end{array}$ & $\begin{array}{l}34,8 \\
38,7 \\
35,0 \\
57,4 \\
28,1 \\
52,6 \\
52,0\end{array}$ & $\begin{array}{r}100 \\
100 \\
100 \\
100 \\
100 \\
100 \\
100\end{array}$ \\
\hline Revenu total & $\begin{array}{l}19999 \text { \$ et moins } \\
20000 \$-39999 \$ \\
40000 \$-59999 \$ \\
60000 \text { \& et plus }\end{array}$ & $\begin{array}{l}22,5 \\
28,7 \\
44,7 \\
47,6\end{array}$ & $\begin{array}{l}19,4 \\
18,6 \\
22,3 \\
21,4\end{array}$ & $\begin{array}{l}58,1 \\
52,7 \\
33,0 \\
31,0\end{array}$ & $\begin{array}{l}100 \\
100 \\
100 \\
100\end{array}$ \\
\hline
\end{tabular}

*Toutes les relations entre les variables présentées dans ce tableau sont statistiquement significatives (le test du khi-carré) au seuil de 0,10 ou moins.

\begin{tabular}{|c|c|c|c|}
\hline \multicolumn{4}{|c|}{ 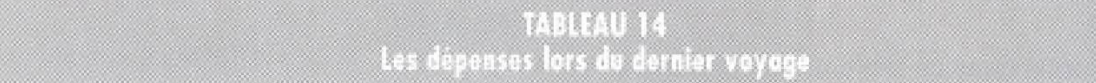 } \\
\hline Dépenses & Fréquence & $\%$ & \% cumulé \\
\hline $\begin{array}{l}100 \text { \$ et moins } \\
101 \$-500 \$ \\
501 \$-2000 \$ \\
2001 \text { \& et plus } \\
\text { T0TAL }\end{array}$ & $\begin{array}{l}168 \\
187 \\
85 \\
37 \\
477\end{array}$ & $\begin{array}{l}35,2 \\
39,2 \\
17,8 \\
7,7 \\
100\end{array}$ & $\begin{array}{l}35,2 \\
74,5 \\
92,3 \\
100\end{array}$ \\
\hline
\end{tabular}


sensiblement avec le niveau de revenus. Dans la catégoric 501 \$a 2000 \$, la coupure se fait entre ceux qui gagnent $39999 \$$ et moins et ceux qui gagnent $40000 \$$ et plus : dans la catégorie $2001 \mathrm{~S}$ et plus, il y a une évolution graduelle des dépenses selon la strate des revenus $(3,3 \%$ pour ceux qui gagnent 19999 set moins et $15,3 \%$ pour ceux qui gagnent 60000 \$ et plus).

\section{L'objectif principal du dernier voyage et avec qui ce dernier voyage a été fait}

Le tableau $\mathbb{\|} 6$ donne l'objectif du dernier voyage. Ce tableau permet de constater que le principal objectif est de rencontrer des parents ou des amis et que 21,2\% des personnes voyagent pour se reposer, $12,5 \%$ pour faire la découverte de nouveaux endroits et $7 \%$ pour obtenir des traitements médicaux.

Le tableau 17 repond à la question: * Avec qui avez-vous fait ce dernier voyage ? $n$

On voit dans ce tableau que. lors du dernier voyage, $67,1 \%$ des personnes voyagent en famille ou en couple, $17,7 \%$ voyagent seules e $15,2 \%$ voyagent avec des amis ou des collègues de travail ou encore avec des groupes organises.

\section{Une étude poussée des relations entre le revenu, le niveau de mobilité et la réalisation d'un voyage dans la dernière année}

Pour mieux comprendre les relations entre le revenu, le niveau de mobilité et la réalisation d'un voyage dans la dernière annec, nous avons fait des croisements à trois variables ; les résultats de ces croisements, présentés au tableau 18 , sont assez ćtonnants.

Nous notons dans ce tableau que le départ en voyage tend à augmenter selon la strate de revenus ainsi que pour les niveaux de mobilité : audition ou vision, mobilite réduite et mobilité très réduite.

L'effet de l'augmentation des revenus ne joue pas (le résultat du khi-carré, avec une

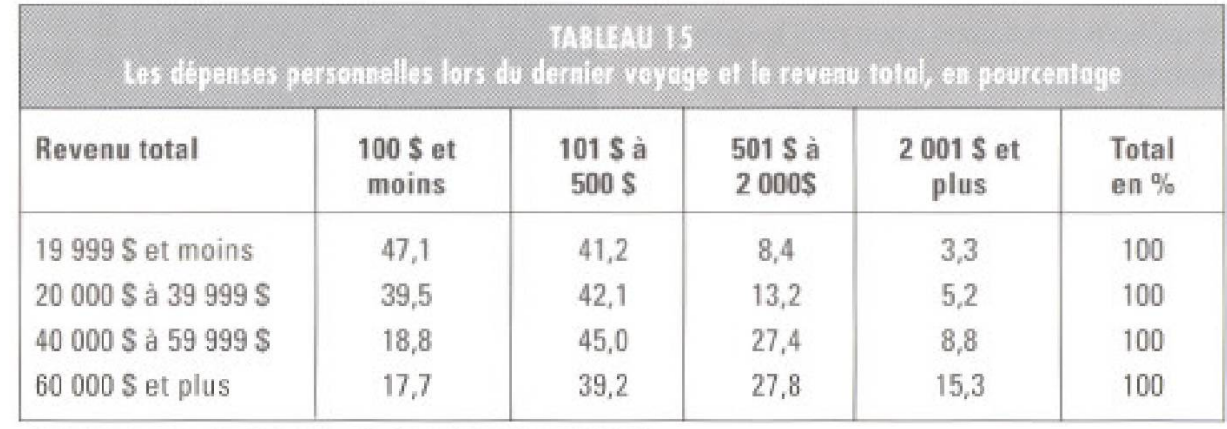

* Khi-carré : 45,49; DL : 9 : Signification : 0,000

\begin{tabular}{|l|c|c|}
\hline \multicolumn{3}{|c|}{ Uobjecti prindipt du tereier voyeys } \\
\hline Objectif principal & Fréquence & $\%$ \\
\hline Parents/amis & 249 & 44,27 \\
Se reposer & 118 & 21,2 \\
Autres raisons & 81 & 14,6 \\
Découverte & 69 & 12,5 \\
Traitements & 39 & 7,0 \\
médicaux & & 100 \\
TOTAL & 557 & \\
\hline
\end{tabular}

\begin{tabular}{|l|c|c|}
\hline \multicolumn{3}{|c|}{ Aver qui s'osi col le derviè roycge ? } \\
\hline Avec qui & Fréquence & $\%$ \\
\hline Seul & 98 & 17,7 \\
En couple & 172 & 30,9 \\
Famille & 201 & 36,2 \\
Amis/collègues/ & & \\
groupes & 85 & 15,2 \\
TOTAL & 556 & 100 \\
\hline
\end{tabular}

\begin{tabular}{|c|c|c|c|c|}
\hline \multicolumn{5}{|c|}{ 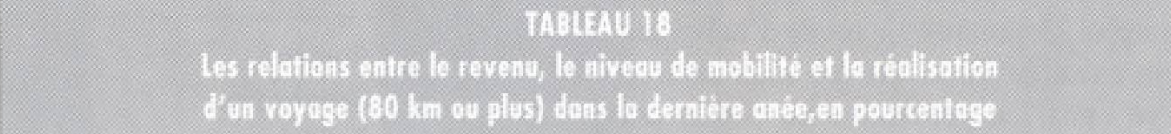 } \\
\hline \multirow[t]{2}{*}{ Mobilité } & \multirow[t]{2}{*}{ Revenu } & \multicolumn{2}{|c|}{$\begin{array}{l}\text { Au moins un voyage de } 80 \mathrm{~km} \text { ou } \\
\text { plus dans la dernière année }\end{array}$} & \multirow[t]{2}{*}{ Total } \\
\hline & & Oui & Non & \\
\hline \multirow[t]{4}{*}{ Audition ou vision* } & 19999 S et moins & 47,8 & 52,2 & 100 \\
\hline & 20000 S-39 9995 & 55,6 & 44,4 & 100 \\
\hline & $40000 \$ 599995$ & 88,2 & 11,8 & 100 \\
\hline & 60000 S et plus & 90,9 & 9,1 & 100 \\
\hline \multirow[t]{4}{*}{ Réduite** } & 19999 s et moins & 46,5 & 53,5 & 100 \\
\hline & $20000 \$-39999 \$$ & 59,6 & 40,4 & 100 \\
\hline & $40000 \$-59999 \$$ & 76,7 & 23.3 & 100 \\
\hline & 60000 S et plus & 72,7 & 27,3 & 100 \\
\hline \multirow[t]{4}{*}{ Très rẻduite ${ }^{* \#}$} & 19999 S et moins & 38,2 & 61,8 & 100 \\
\hline & 20000 s-39 999 s & 40,9 & 59,1 & 100 \\
\hline & 40000 \$-59 999 \$ & 68,4 & 31,6 & 100 \\
\hline & 60000 s et plus & 70,6 & 29,4 & 100 \\
\hline \multirow[t]{4}{*}{$\begin{array}{l}\text { Extrêmement } \\
\text { réduite }\end{array}$} & 19999 S et moins & 45,5 & 54,5 & 100 \\
\hline & $20000 \$-39999 \$$ & 56,7 & 43,3 & 100 \\
\hline & 40000 \$-59 $999 \mathrm{~s}$ & 43,5 & 56,5 & 100 \\
\hline & 60000 \$ et plus & 63,6 & 56,5 & 100 \\
\hline
\end{tabular}

\footnotetext{
Khi-carré : 10,$85 ;$ DL : 3 ; Signification : 0,013

* Khi-carré : 24,$68 ;$ DL : 3 ; Signification : 0,000

*** Khi=carre : 9,$65 ; 0 L: 3$; Signification : 0,022

**** Khil-carré : 2,$98 ; 0 L: 3 ;$ Signification : 0,394
} 


\begin{tabular}{|c|c|c|}
\hline \multicolumn{3}{|c|}{ 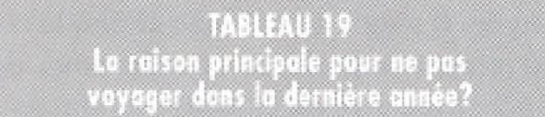 } \\
\hline Raison principale & Fréquence & $\%$ \\
\hline Raisons de santé & 151 & 33,9 \\
\hline Condition physique & 101 & 22,7 \\
\hline Raisons économiques & 62 & 14,0 \\
\hline Autres raisons & 131 & 29,4 \\
\hline TOTAL & 445 & 100 \\
\hline
\end{tabular}

signification de 0,394 , en témoigne) dans le cas des personnes à mobilité cxtrềmement réduite (et cela malgré les différences observées dans les pourcentages).

\section{Les principales raisons pour ne pas voyager dans la dernière année}

Le tableau 19 donne les principales raisons évoquées pour ne pas voyager dans la dernière année.

On voit ici que les raisons de santé et la condition physique regroupent $56,6 \%$ des répondants. Les raisons économiques sont citées par $14 \%$ des personnes interrogées et d'autres raisons par $29,4 \%$.

Le tableau 20 présente les relations entre les raisons principales pour ne pas voyager el quatre variables socio-économiques.

Les raisons de santé et la condition économique sont les raisons les plus importantes (en pourcentage) quel que soit le niveau de mobilité. Les personnes ayant des problèmes d'audition ou de vision et les personnes à mobilité réduite sont les plus nombreuses $(20 \%$ et $18,4 \%$ respectivement) à mentionner des problèmes économiques comme obstacles à voyager.

Les raisons de santé augmentent (en pourcentage) avec les strates d'âge : $19,2 \%$ chez les 24 ans et moins et $40,6 \%$ dans le groupe des 65 ans et plus, Les raisons économiques sont plus importantes chez les 24 ans et moins et dans la strate des $35=$ 44 ans. Les plus jeunes sont aussi les plus nombreux à citer d'autres raisons (que celles proposées) pour ne pas voyager.

Ce sont, à la fois, les moins scolarisés et les plus scolarisés qui evoquent, avec un

\begin{tabular}{|c|c|c|c|c|c|c|}
\hline \multicolumn{7}{|c|}{ 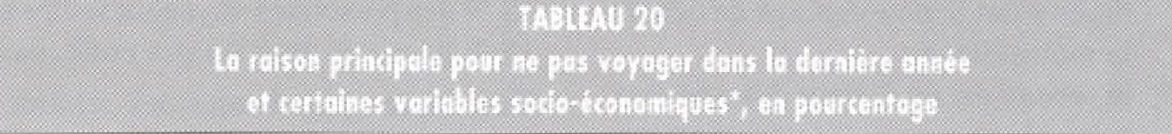 } \\
\hline & Variables & $\begin{array}{l}\text { Raisan } \\
\text { de santé }\end{array}$ & $\begin{array}{l}\text { Conditions } \\
\text { physique }\end{array}$ & $\begin{array}{c}\text { Raisons } \\
\text { ecunomiques }\end{array}$ & $\begin{array}{l}\text { Autres } \\
\text { raisons }\end{array}$ & Total \\
\hline \multirow[t]{4}{*}{ Mobilité } & Audition ou vision & 20,0 & 20,0 & 20,0 & 40,0 & 100 \\
\hline & Mobilité réduite & 33,1 & 19,7 & 18,4 & 28,8 & 100 \\
\hline & Mobilité très réduite & 39,8 & 28.0 & 7,5 & 24,7 & 100 \\
\hline & Mobilité extrêment réduite & 35,6 & 25,3 & 6,9 & 32,2 & 100 \\
\hline \multirow[t]{6}{*}{ Áge } & 24 ans et moins & 19,2 & 11,5 & 23,1 & 46,2 & 100 \\
\hline & $25-34$ ans & 21,1 & 31,6 & 15.8 & 31,5 & 100 \\
\hline & $35-44$ ans & 16,3 & 20,4 & 36,7 & 26,6 & 100 \\
\hline & $45-54$ ans & 33,8 & 29,9 & 10,3 & 26,0 & 100 \\
\hline & $55-64$ ans & 36,6 & 14,6 & 17,1 & 31,7 & 100 \\
\hline & 65 ans et plus & 40,6 & 24,5 & 7,3 & 27,6 & 100 \\
\hline \multirow[t]{4}{*}{ Scolarité } & Primaire & 35,6 & 23,0 & 17,2 & 24,2 & 100 \\
\hline & Secondaire & 29,0 & 23,4 & 139 & 33,7 & 100 \\
\hline & Collégial & 33,8 & 35,4 & 9,2 & 21,6 & 100 \\
\hline & Universitaire & 50,0 & 5,6 & 18,5 & 25,9 & 100 \\
\hline \multirow[t]{7}{*}{ Occupation } & $\begin{array}{l}\text { Professionnels/ } \\
\text { gestionnaires }\end{array}$ & 14,3 & 28,6 & 28,6 & 28,5 & 100 \\
\hline & Services & 13,0 & 21,7 & 17,4 & 47,9 & 100 \\
\hline & Techniciens/ouvriers & 28,6 & 23,8 & 19,0 & 28,6 & 100 \\
\hline & Au foyer & 31,2 & 24,7 & 20,8 & 23,3 & 100 \\
\hline & Etudiants & 11,8 & 11,8 & 29,4 & 47,0 & 100 \\
\hline & Retraités & 41,5 & 21,2 & 9,2 & 28,1 & 100 \\
\hline & Sans emploi & 29,8 & 26,2 & 14,3 & 29,7 & 100 \\
\hline
\end{tabular}

* Toutes les relations entre les variables présentées dans ce tableau sont statistiquement significatives (le test du khi-carré) au seuil de 0,10 ou moins.

pourcentage plus élevé, des raisons économiques pour expliquer qu'ils ne partent pas en voyage. Les raisons de santé sont plus nombreuses chez les retraites $(4 I, 5 \%)$ et les raisons economiques plus fortes, en pourcentage, chez les professionnels et les gestionnaires et chez les étudiants. La catégorie autres raisons est très élevée chez les personnes qui travaillent dans les services et chez les étudiants.

\section{Conclusion}

Il est possible de résumer certains résultats obtenus dans cette enquête :

- Le taux de départ des personnes à capacité physique restreinte est de $55,7 \%$.

- Ce pourcentage des départs varie sensiblement en fonction des principales variables socio-économiques. Le por- trait des \& non partants $x$ est assez falcile at tracer, ce sont :

1. des personnes à mobilité très réduite ou extrêmement réduite :

2. il y a un léger écat entro les sexes: les hommes voyagent un peu plus que les femmes:

3. les pourcentages des départs déclinent avec l'âge (il est de $67 \%$ pour les 25 ans et moins et de $48 \%$ pour les 65 ans et plus);

4. le pourcentage est plus faible au Québec et chez les personnes de langue maternelle française ;

5. les personnes qui ont une scolarité primaire ou secondaire voyagent moins que les personnes qui possèdent un niveau supérieur de scolarité :

6. les «actifs $\%$ voyagent plus que les * inactifs * les personnes *au 
foyer so ont le pourcentage de départ le moins élevé :

7. le revenu est un facteur déterminant en ce qui concerne les pourcentages de départ en vacances; il y a une nette différence dans les pourcentages de départ entre ceux qui gagnent $39999 \$$ et moins et ceux qui gagnent $40000 \$$ et plus.

- Les personnes à capacité physique restreinte qui voyagent le font beaucoup puisque la moyenne des voyages réalisés, dans la dernière année, est de quatre ; mais la dispersion autour de la moyenne est élevée car le coefficient de variation est de $67 \%$. Le nombre des voyages réalisés est en partie déterminé par la langue maternelle, la scolarité, l'occupation principale et le revenu.

- La moyenne de la durée des séjours lors du dernier voyage est de huit jours (le coefficient de variation est de $65 \%$ ). La duree des vacinces est surtout influencé par l'âge et l'occupation principale.

- La plupart des personnes interrogées voyagent dans leur propre région ; $28 \%$ d'entre elles voyagent à l'étranger ( $15 \%$ aux États-Unis et $13 \%$ dans d'autres pays). La destination choisic est influencée par la plupart des variables socioéconomiques.

- Les principaux moyens de transport sont l'automobile $(63 \%)$ et l'avion (19\%).

- Le principal mode d'hébergement est le séjour chez des parents et des amis $(47 \%)$; l'hôtel-motel arrive en deuxicme position (31 \%). Les autres modes privés d'hébergement se partagent les $22 \%$ restants.

- La moyenne des dépenses lors du dernier voyage est de $714 \$$ (il faut noter que le coefficient de variation est de $64 \%$ ) : malgre cette moyenne, il faut tenir compte du fait que $72 \%$ des personnes interrogées ont dépensé 500 S et moins lors de leur dernier voyage.

- Parmi les personnes interrogées, $45 \%$ voyagent pour visiter des parents et des amis, $21 \%$ pour se reposer, $12 \%$ pour faire du tourisme et $7 \%$ pour suivre des traitements médicaux.

La plupart des personnes voyagent en famille $(36 \%)$ ou en couple $(31 \%)$;
$18 \%$ des personnes voyagent seules et $15 \%$ avec des amis ou des collègues.

- Une étude approfondie des relations entre le niveau de mobilité, le revenu el la réalisation d'un voyage montre que les départs en voyage, pour les personnes à mobilité extrêmement réduité, ne sont pas influencés, comme dans les autres groupes, par des augmentations du revenu familial. Ce résultat tend à démontrer que des obstacles importants se dressent, pour cette catégorie de répondants, face au désir de voyager.

Sur le plan socio-économique, la population des personnes à capacité physique restreinte est doublement marginalisée. Au handicap physique s'ajoute souvent la précarité économique qui est, elle-même, la résultante d'une scolarité faible et d'un nombre éleve d'inactifs.

Malgré cela, dans l'ensemble, la population des personnes à capacité physique restreinte voyage presque autant que la population globale car les taux de départ. toutes catégories socio-économiques confondues, tournent autour de $50 \%$. Généralement, les personnes ă capacité physique restreinte qui ont surmonté les obstacles inhérents au départ en voyage ont tendance à voyager fréquemment et de façon pro- longée. Il s'agit donc d'une clientèle relativement importante et méconnue de l'industrie du voyage. Nous l'évaluons à environ $6 \%$ a $8 \%$ de la population canadienne. Il faut néanmoins souligner que la moyenne de leurs dépenses est moins élevée que celle des autres groupes de voyageurs.

\section{Notes}

1 Le present article est tiré d'un rapport de recherche (à paraître) portant sur a Un marché $\mathrm{d}$ "avenir. Les comportements touristiques des personnes à capacité physique restreinte au Canada $*$; celte étude est financée par Keroul, organisme qui a pour mission de rendre le tourisme accessible aux personnes a capacité physique restreinte.

2 Les cas admissibles comprennent seulement les handicaps physiques: , les deficiences intellectuelles, les maladies psychologiques et psychosomatiques n ont pas été considerées dans cette étude.

3 Enquête sur les voyages des Canadiens. Voyages interieurs en 1998 (1999), Catalogue no 87-212-XIF, Statistique Canada, p. 21.

4 II indique l"absence de relation entre les deux variables.

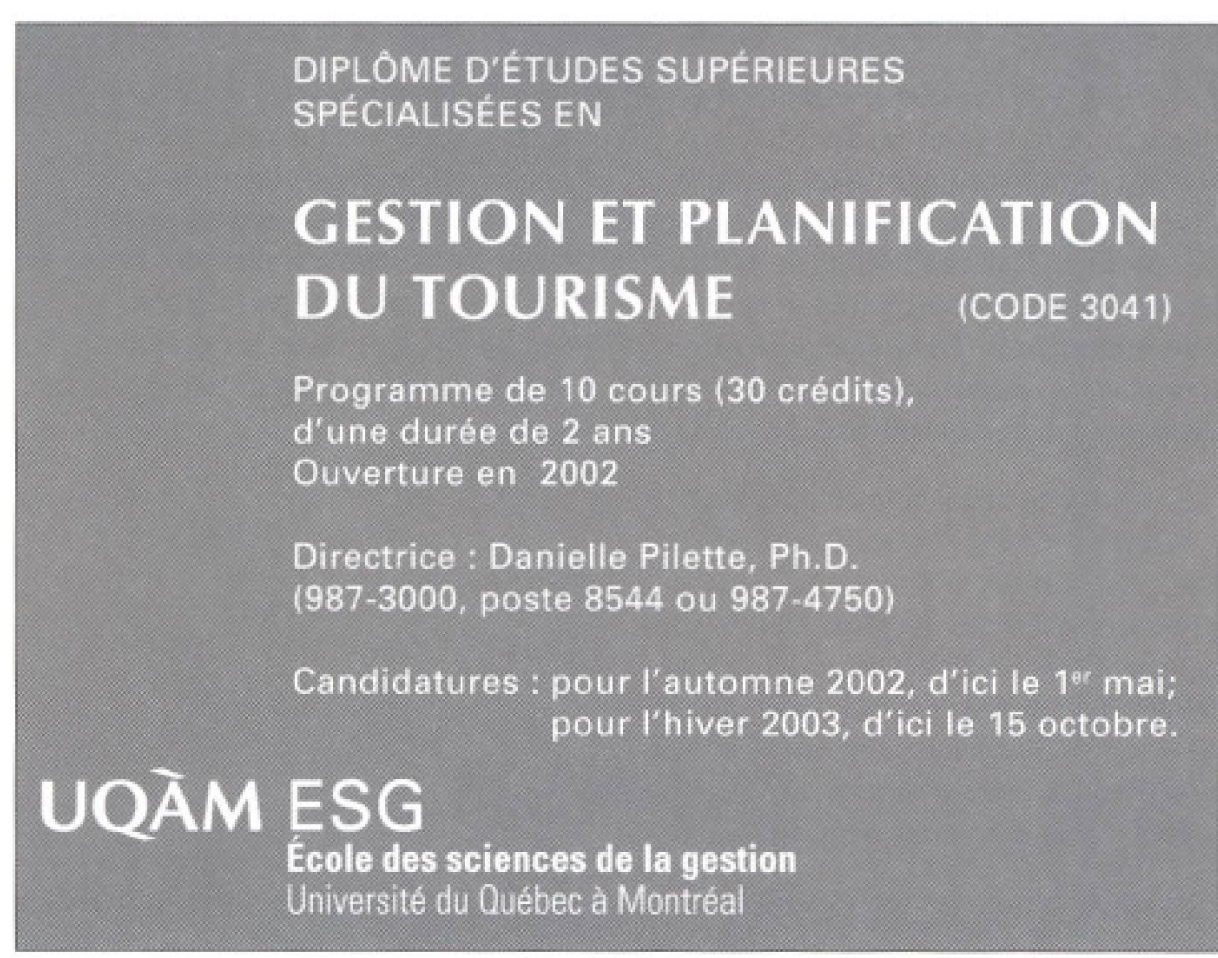

DIPLOME D'ÉTUDES SUPÉRIEURES SPECIALISÉES EN

\section{GESTION ET PLANIFICATION DU TOURISME \\ ICODE 3041}

Programme de 10 cours $(30$ crédits)

d'une durée de 2 ans

Ouverture en 2002

Directrice : Danielle Pilette, Ph.D.

$(987-3000$, poste 8544 ou $987-4750)$

Candidatures : pour l'automne 2002, $\mathrm{d}^{\mathrm{T}}$ 'ci le $1^{\mathrm{t}} \mathrm{mai}$; pour I'hiver 2003 , d'ici le 15 octobre. 